TRANSACTIONS OF THE

AMERICAN MATHEMATICAL SOCIETY

Volume 351, Number 12, Pages 4947-4961

S 0002-9947(99)02487-3

Article electronically published on August 10, 1999

\title{
BEHAVIOR NEAR THE BOUNDARY OF POSITIVE SOLUTIONS OF SECOND ORDER PARABOLIC EQUATIONS. II
}

\author{
E. B. FABES, M. V. SAFONOV, AND YU YUAN
}

\begin{abstract}
A boundary backward Harnack inequality is proved for positive solutions of second order parabolic equations in non-divergence form in a bounded cylinder $Q=\Omega \times(0, T)$ which vanish on $\partial_{x} Q=\partial \Omega \times(0, T)$, where $\Omega$ is a bounded Lipschitz domain in $\mathbb{R}^{n}$. This inequality is applied to the proof of the Hölder continuity of the quotient of two positive solutions vanishing on a portion of $\partial_{x} Q$.
\end{abstract}

\section{INTRODUCTION}

In this paper we are concerned with the boundary behavior of positive solutions $u$ and $v$ of the parabolic non-divergence equation

$$
L u=\sum_{i, j=1}^{n} a_{i j}(x, t) D_{i j} u(x, t)-D_{t} u(x, t)=0
$$

near an open portion of the Lipschitz lateral boundary where $u$ and $v$ are assumed to vanish. We prove that $\frac{v}{u}$ is locally Hölder continuous up to that portion of the lateral boundary (Theorem 4.6 and related results in Sec. 4.3).

In our proof of the Hölder continuity of the quotient we first derive so called boundary backward Harnack inequality (Theorem 3.7) which is of considerable interest in itself. It states that any non-negative solution of (1) in a bounded cylinder $Q=\Omega \times(0, T)$, which vanishes on the entire lateral boundary $\partial \Omega \times(0, T)$, satisfies

$$
u(x, s) \leq N u(x, t)
$$

uniformly for all $(x, t) \in Q$, such that $s \geq t \geq s-d^{2} \geq \delta^{2}=$ const $>0$, with $N$ independent of $u$, where $d=\operatorname{dist}(x, \partial \Omega)$. Note that (2) is different from the usual Harnack inequality (5), which is formulated in Theorem 2.2 below; here we have $u(Y) \leq N u(X)$ with $X=(x, t), Y=(x, s)$.

The above two results in parabolic divergence case were proved in a recent paper [FS]. The boundary backward Harnack inequality (2) was first obtained in [G] and [FGS] for the non-divergence and divergence cases when the coefficients are timeindependent. Notice that the constant $N$ in (2) does not depend on $d=\operatorname{dist}(x, \partial \Omega)$. A weaker estimate with $N$ depending also on $d$ is called the interior backward Harnack inequality. In combination with the usual Harnack inequality (5), this

Received by the editors August 4, 1997.

1991 Mathematics Subject Classification. Primary 35K10, 35B05; Secondary 35B45, 31B25.

Key words and phrases. Harnack inequality, Hölder continuity, caloric measure.

The second and third authors are partially supported by NSF Grant No. DMS-9623287.

(C)1999 American Mathematical Society 
inequality (2) implies the elliptic-type Harnack inequality (Theorem 3.6), which was proved in $[\mathrm{G}]$ for the non-divergence case and in [FGS] for the divergence case. The Hölder continuity of the quotient for two positive solution of heat equation $\triangle u$ $-u_{t}=0$ was proved in [ACS]. The corresponding results for the (non-divergence and divergence) elliptic equation is contained in $[\mathrm{B}],[\mathrm{AC}]$ and $[\mathrm{FGMS}]$. When the domain $\Omega$ is smooth, the estimate (2) for non-divergence can be extended for all $s \geq t \geq \delta^{2}$.

The results and methods of this paper are independent of [FS], though the structure of these two papers is similar. Moreover, our approach provides an alternative proof of the Hölder continuity of quotients in [ACS], [FS] for the parabolic divergence case and also [JK], [B], [AC], [FGMS] for the elliptic case, where it was derived via the estimates for the Green's functions and the doubling property for the corresponding $L$-caloric ( $L$-harmonic) measures. The examples in $[\mathrm{FK}]$ and $[\mathrm{S}]$ show that the appropriate estimates for Green's functions in the non-divergence case fail. Regarding the doubling property in the parabolic divergence case, it follows automatically from the backward Harnack inequality (see [FGS]); for the non-divergence case it is proved in a forthcoming paper [SY].

Some intermediate results here (Statements 3.1-3.6) are basically contained in $[\mathrm{G}]$. We give their simplified proofs for completeness of presentation.

In this paper, we assume the coefficients $a_{i j}(x, t)$ are measurable and for all $X=(x, t) \in \Omega \times(0, \infty), \xi \in R^{n}$,

$$
\nu|\xi|^{2} \leq \sum_{i, j=1}^{n} a_{i j}(X) \xi_{i} \xi_{j} \leq \nu^{-1}|\xi|^{2}
$$

with a constant $\nu \in(0,1]$. However, by means of appropriate approximation procedures, all our estimates for solutions can be reduced to the similar estimates with smooth $a_{i j}$ and $u$. It is important only that these estimates do not depend on the smoothness of $a_{i j}$ and $u$. So we may assume that all the functions $a_{i j}$ and $u$ in (1) are smooth.

The preliminary draft of the paper was ready before Professor E. B. Fabes passed away unexpectedly. We hope the present paper meets his high standards, though we are responsible for this final version.

Acknowledgments. The authors would like to thank Professor N. N. Ural'tseva for her useful remarks and suggestions.

\section{Assumptions And Known Results}

For an arbitrary domain $V \subset \mathbb{R}^{n+1}$, we define its parabolic boundary $\partial^{\prime} V$ as the set of all points $X \in \partial V$ such that there is a continuous curve lying in $V \cup\{X\}$ with initial point $X$, along which $t$ is non-decreasing. In particular, for $Q=\Omega \times(0, T)$ we have

$$
\partial^{\prime} Q=\partial_{x} Q \cup \partial_{t} Q
$$

where the lateral boundary $\partial_{x} Q=\partial \Omega \times(0, T)$, and $\partial_{t} Q=\bar{\Omega} \times\{0\}$.

The following comparison principle is well-known.

Theorem 2.1. Let $V$ be a bounded domain in $\mathbb{R}^{n+1}, u, v \in C^{2}(V) \cap C(\bar{V}), L u \leq$ $L v$ in $V$, and $u \geq v$ on $\partial^{\prime} V$. Then $u \geq v$ on $\bar{V}$. 
For $X=(x, t) \in \mathbb{R}^{n+1}$ and $r>0$, a "standard" cylinder

$$
C_{r}(X)=C_{r}(x, t)=B_{r}(x) \times\left(t-r^{2}, t\right),
$$

where $B_{r}(x)=\left\{y \in \mathbb{R}^{n}:|y-x|<r\right\}$. For $\delta=$ const $>0, \Omega \subset \mathbb{R}^{n}, Q=\Omega \times(0, T)$ we set

$$
\begin{aligned}
& \Omega^{\delta}=\{x \in \Omega: \operatorname{dist}(x, \partial \Omega)>\delta\}=\left\{x \in \Omega: \bar{B}_{\delta}(x) \subset \Omega\right\}, \\
& Q^{\delta}=\Omega^{\delta} \times\left(\delta^{2}, T\right)=\left\{X \in Q: \bar{C}_{\delta}(X) \subset Q\right\} .
\end{aligned}
$$

Theorem 2.2 (Harnack Principle). Let $u$ be a nonnegative solution of Lu=0 in a bounded $Q=\Omega \times(0, T), \delta=$ const $>0$ such that $\Omega^{\delta}$ is a connected set, $X=(x, t), Y=(y, s) \in Q^{\delta}$, and $s-t \geq \delta^{2}$. Then

$$
u(X) \leq N u(Y)
$$

where the constant $N=N(n, \nu$, diam $\Omega, T, \delta)$. For cylinders $Q=C_{r}, r>0$, the constant $N=N\left(n, \nu, \frac{\delta}{r}\right)$.

This theorem was proved in [KS], see also [K, Chap. 4], for the divergence case it was proved in [M1], [M2], see also [FSt].

As in [FGS] and [FS], we assume that a bounded domain $\Omega \subset \mathbb{R}^{n}$ satisfies the following Lipschitz condition with some positive constants $r_{0}$ and $m$.

Assumptions: For each $y \in \partial \Omega$, there is an orthonormal coordinate system centered at $y$ such that

$$
\Omega \cap B_{r_{0}}(y)=\left\{x=\left(x^{\prime}, x_{n}\right): x^{\prime} \in \mathbb{R}^{n-1}, x_{n}>\varphi\left(x^{\prime}\right),|x|<r_{0}\right\},
$$

where $\|\nabla \varphi\|_{\mathfrak{L} \infty} \leq m$.

In such local coordinates, $y \in \partial \Omega$ is represented as $(0,0)$ and $(0, r) \in \Omega$ for all $r \in\left(0, r_{0}\right]$. For $Q=\Omega \times(0, T), Y=(y, s)=(0,0, s) \in \partial_{x} Q=\partial \Omega \times(0, T)$, and $r>0$, we set

$$
\bar{Y}_{r}=\left(0, r, s+r^{2}\right), \quad \underline{Y}_{r}=\left(0, r, s-2 r^{2}\right) .
$$

Throughout this paper, $N$ denotes various positive constants depending only on the original quantities.

\section{BACKWARD HaRnACK INEQUALITIES}

\subsection{Estimates of Solutions Near the Boundary.}

Lemma 3.1. Let $u$ be a nonnegative solution of $L u=0$ in $Q=\Omega \times(0, T)$. Then for any $Y=(y, s) \in \partial_{x} Q$ and $0<r \leq \frac{1}{2} \min \left(r_{0}, \sqrt{T-s}\right)$, we have

$$
M=\sup _{Q_{2 r}} d^{\gamma} u \leq N r^{\gamma} u\left(\bar{Y}_{r}\right)
$$

where $Q_{2 r}=Q \cap C_{2 r}(Y), \quad d=d(X)=\sup \left\{\rho>0: C_{\rho}(X) \subset Q_{2 r}\right\}$, and $\gamma, N$ are positive constants depending only on $n, \nu, m$.

Proof. We fix a point $X \in Q_{2 r}$. By simple geometrical considerations one can see that there exists a finite sequence $\left\{X^{(i)}=\left(x^{(i)}, t^{(i)}\right): i=0,1, \cdots, k\right\} \subset Q$ such that

$$
\begin{gathered}
X^{(0)}=X, \quad X^{(k)}=\bar{Y}_{r}, \quad d_{i} \geq \delta_{0} q^{i} d_{0}, \\
C_{\delta d_{i}}\left(X^{(i-1)}\right) \subset C_{d_{i}}\left(X^{(i)}\right) \subset Q, \quad t^{(i)}-t^{(i-1)} \geq \delta^{2} d_{i}^{2},
\end{gathered}
$$


for all $i=1,2, \cdots, k$, where $d_{i}=\sup \left\{\rho>0: C_{\rho}\left(X^{(i)}\right) \subset Q\right\}, i \geq 0$, and constants $\delta_{0}, \delta \in(0,1), q>1$ depend only on $m$. From (7) it follows

$$
d=d(X) \leq d_{0} \leq \delta_{0}^{-1} q^{-k} d_{k} \leq \delta_{0}^{-1} q^{-k} r .
$$

Further, by Theorem 2.2 we have $u\left(X^{(i-1)}\right) \leq N u\left(X^{(i)}\right), i=1, \cdots, k$, where $N=N(n, \nu, m)>1$. We represent this constant $N$ in the form $N=q^{\gamma}$. Then

$$
u(X)=u\left(X^{(0)}\right) \leq q^{k \gamma} u\left(X^{(k)}\right)=q^{k \gamma} u\left(\bar{Y}_{r}\right) .
$$

Together with (9) this yields $d^{\gamma} u(X) \leq N r^{\gamma} u\left(\bar{Y}_{r}\right)$. Since $X$ is an arbitrary point in $Q_{2 r}$, we arrive at the desired estimate (6).

Lemma 3.2. Let $Q=\Omega \times(0, T), Y=(y, s) \in \partial_{x} Q$, and $0<r \leq \frac{1}{2} \min \left(r_{0}, \sqrt{s}\right)$ be fixed, and let $u$ be a nonnegative solution of $L u=0$ in $Q_{2 r}=Q \cap C_{2 r}(Y)$. Then

$$
u\left(\underline{Y}_{r}\right) \leq N r^{\gamma} \inf _{Q_{r}} d^{-\gamma} u,
$$

where $d=d(x)=\operatorname{dist}(x, \partial \Omega)$ for $X=(x, t) \in Q_{r}=Q \cap C_{r}(Y)$, and $\gamma, N$ are positive constants depending only on $n, \nu, m$.

Proof. It follows the lines of the proof of the previous lemma, only we replace $\bar{Y}_{r}$ by $\underline{Y}_{r}$ in (7), and instead of (8) we now take

$$
C_{\delta d_{i}}\left(X^{(i+1)}\right) \subset C_{d_{i}}\left(X^{(i)}\right) \subset Q, t^{(i)}-t^{(i+1)} \geq \delta^{2} d_{i}^{2} .
$$

Then we have (9) and

$$
u\left(\underline{Y}_{r}\right)=u\left(X^{(k)}\right) \leq q^{k \gamma} u\left(X^{(0)}\right)=q^{k \gamma} u(X) \leq N r^{\gamma} d^{-\gamma} u(X),
$$

which proves (10).

The next theorem is a boundary Harnack inequality. Such kind of estimate is also referred to as Carleson type inequality. The estimate (12) was first proved by S. Salsa in [Sl] (Theorem 3.1) for divergence case, and by N. Garofalo in [G] (Theorem 2.3) for non-divergence case.

Theorem 3.3. Let $Y=(y, s) \in \partial_{x} Q$ and $0<r \leq \frac{1}{2} \min \left(r_{0}, \sqrt{T-s}, \sqrt{s}\right)$ be fixed. Then for any nonnegative solution of $L u=0$ in $Q$, which continuously vanishes on $\Gamma=\partial_{x} Q \cap C_{2 r}(Y)$, we have

$$
M_{0}=\sup _{Q_{2 r}} d_{0}^{\gamma} u \leq N r^{\gamma} u\left(\bar{Y}_{r}\right)
$$

where

$$
d_{0}=d_{0}(X)=\sup \left\{\rho>0: C_{\rho}(X) \subset C_{2 r}(Y)\right\},
$$

and $\gamma, N$ are positive constants depending only on $n, \nu, m$. In particular,

$$
\sup _{Q_{r}} u \leq N u\left(\bar{Y}_{r}\right) .
$$

First we prove the following elementary estimate; such kinds of estimates usually serve as intermediate steps in the proof of boundary Hölder estimates (in the divergence case, see [LSU, Chap. II], Sec. 8, and [T, Sec. 4]). 
Lemma 3.4. Let a domain $U \subset C_{2 r}=C_{2 r}(Y)$, where $r>0$ and $Y=(y, s) \in$ $\mathbb{R}^{n+1}$. Let $Z=(z, \tau)$ and $0<\varepsilon \leq 1$ be such that

$$
B_{\varepsilon r}(z) \times\{\tau\} \subset C_{2 r} \backslash U, \quad s-4 r^{2}<\tau \leq s-2 r^{2} .
$$

Then for any $u$ satisfying $L u \geq 0$ in $U, \quad u \leq 0$ on $\left(\partial^{\prime} U\right) \backslash\left(\partial^{\prime} C_{2 r}\right)$, and $\sup _{U} u>0$, we have

$$
\sup _{U \cap C_{r}} u \leq \theta \sup _{U} u
$$

with a constant $\theta=\theta(n, \nu, \varepsilon) \in(0,1)$.

Proof. We fix $X_{0}=\left(x_{0}, t_{0}\right) \in U \cap C_{r}$. Without loss of generality we may assume $\sup _{U} u=1, r=1$, and $Z=(z, \tau)=(0,0)$. Then from (13) it follows that $\left|x_{0}\right| \leq 3$, $1 \leq t_{0} \leq 4$. Consider the function $v=e^{-\lambda t} w^{2}$, where $w=\varepsilon^{2}-|x-t l|^{2}, l=x_{0} / t_{0}$, and $\lambda=$ const, on the slant cylinder

$$
V=\left\{(x, t):|x-t l|<\varepsilon, \quad 0<t<t_{0}\right\} .
$$

We have

$$
L v=e^{-\lambda t}\left(\lambda w^{2}+2 w L w+F\right), \text { where } F=2 \sum_{i, j} a_{i j} D_{i} w D_{j} w \geq 2 \nu|D w|^{2}
$$

and $|L w| \leq N=N(n, \nu)$ in $V$. Since $F \geq \nu \varepsilon^{2}$ and $w$ is small near

$$
\partial^{\prime} V=\left\{(x, t):|x-t l|=\varepsilon, 0<t<t_{0}\right\},
$$

there exists $\varepsilon_{1}=\varepsilon_{1}(n, \nu, \varepsilon) \in(0, \varepsilon)$ such that $L v \geq 0$ for $\varepsilon_{1} \leq|x-t l| \leq \varepsilon$, $0 \leq t \leq t_{0}$, and arbitrary $\lambda \geq 0$. On the remaining part of $U$, we also have $L v \geq 0$, provided $\lambda=\lambda(n, \nu, \epsilon)>0$ is large enough.

Further, the parabolic boundary $\partial^{\prime}(U \cap V)=\Gamma_{1} \cup \Gamma_{2}$ where $\Gamma_{1} \subset \partial_{x} V \cap \bar{U}$, $\Gamma_{2}=\left(\partial^{\prime} U \backslash \partial^{\prime} C_{2}\right) \cap V$. Since $u \leq \sup _{U} u \leq 1, v=0$ on $\Gamma_{1}$ and $u \leq 0, v \leq \varepsilon^{4} \leq 1$ on $\Gamma_{2}$, we have $u+v \leq 1$ on $\partial^{\prime}(U \cap V)$. Moreover, $L(u+v) \geq 0$ in $U \cap C_{2 r}$. By the comparison principle, $u+v \leq 1$ in $\overline{U \cap V}$. Hence

$$
u\left(X_{0}\right) \leq 1-e^{-4 \lambda} \varepsilon^{4}=\theta=\theta(n, \nu, \varepsilon) \in(0,1) .
$$

Since $X_{0} \in U \cap C_{2 r}$ is arbitrary, we get the estimate (14).

By iterating the estimate (14), we get the following Hölder estimate (cf. [G, Lemma 2.1]).

Corollary 3.5. Under the assumption of Theorem 3.3, for $\rho \leq r$, we have

$$
\sup _{Q_{\rho}} u \leq 2^{\alpha}\left(\frac{\rho}{r}\right)^{\alpha} \sup _{Q_{r}} u
$$

where $\alpha=\alpha(n, \nu, m)=-\log _{2} \theta>0$.

Proof of Theorem 3.3. Comparing (11) with (6), we see that it suffices to prove the estimate

$$
M_{0} \leq N_{0} M, \text { with } N_{0}=N_{0}(n, \nu, m) .
$$

We choose $\varepsilon_{0}=\varepsilon_{0}(n, \nu, m) \in\left(0, \frac{1}{3}\right)$ small enough, so that

$$
\theta_{0}=\left(1-3 \varepsilon_{0}\right)^{-\gamma} \theta<1 \text {, }
$$

where $\theta<1$ is the constant in Lemma 3.4. For arbitrary $X=(x, t) \in Q_{2 r}$, we consider separately two possible cases (a) and (b). 
(a) $d=d(X) \leq \varepsilon_{0} d_{0}(X)$. In this case, $d=\operatorname{dist}(x, \partial \Omega)=\left|x-x_{0}\right|$ for some $x_{0} \in \partial \Omega$. By Lemma 3.4 applied to $u$ in $Q_{2 d}\left(X_{0}\right)=Q \cap C_{2 d}\left(X_{0}\right), X_{0}=\left(x_{0}, t\right)$, we have

$$
u(X) \leq \sup _{Q_{d}\left(X_{0}\right)} u \leq \theta \sup _{Q_{2 d}\left(X_{0}\right)} u
$$

Further, $d_{0}(X) \leq d_{0}(Z)+|X-Z|$, where

$$
|X-Z|=\max \left(|x-z|,|t-\tau|^{\frac{1}{2}}\right) \leq 3 d \leq 3 \varepsilon_{0} d_{0}(X)
$$

for arbitrary $Z=(z, \tau) \in Q_{2 d}\left(X_{0}\right)$. Therefore, $\left(1-3 \varepsilon_{0}\right) d_{0}(X) \leq d_{0}(Z)$ for such $Z$, and together with (17), (16), (11), this gives us

$$
d_{0}^{\gamma} u(X) \leq\left(1-3 \varepsilon_{0}\right)^{-\gamma} \theta \sup _{Q_{2 d}\left(X_{0}\right)} d_{0}^{\gamma} u \leq \theta_{0} M_{0} .
$$

(b) $d=d(X)>\varepsilon_{0} d_{0}(X)$. Obviously, in this case,

$$
d_{0}^{\gamma} u(X) \leq \varepsilon_{0}^{-\gamma} d^{\gamma} u(X) \leq N_{0} M \text { with } N_{0}=\varepsilon_{0}^{-\gamma} .
$$

Combining (18) and (19), we now have

$$
M_{0}=\sup _{Q_{2 r}} d_{0}^{\gamma} u \leq \max \left(\theta_{0} M_{0}, N_{0} M\right)=N_{0} M,
$$

because $\theta_{0}<1$. So the estimate (15) is proved.

3.2. Backward Harnack Inequalities. The following elliptic-type Harnack inequality is similar to Theorem 2.6 in [G] (see also [FGS], Theorem 1.3, for the divergence case).

Theorem 3.6. Let $u$ be a nonnegative solution of $L u=0$ in $Q=\Omega \times(0, T)$ which continuously vanishes on $\partial_{x} Q$, and let $0<\delta \leq \frac{1}{2} \min \left(r_{0}, \sqrt{T}\right)$. Then there exists a positive constant $N=N\left(n, \nu, m, r_{0}, \operatorname{diam} \Omega, T, \delta\right)$, such that

$$
\sup _{Q^{\delta}} u \leq N \inf _{Q^{\delta}} u
$$

where $Q^{\delta}$ is defined in (4).

Proof. Applying the maximum principle, the boundary Harnack inequality (Theorem 3.3) and the Harnack principle (Theorem 2.2), we have

$$
\sup _{Q^{\delta}} u \leq \sup _{x \in \Omega} u\left(x, \frac{\delta^{2}}{4}\right) \leq N_{1} \sup _{x \in \Omega^{\mu \delta}} u\left(x, \frac{\delta^{2}}{2}\right) \leq N \inf _{Q^{\delta}} u
$$

where $\mu=\mu(m)>0, N_{1}=N_{1}(n, \nu, m)$, and $N=N\left(n, \nu, m, r_{0}\right.$, diam $\left.\Omega, T, \delta\right)$.

The boundary backward Harnack inequality is formulated as follows.

Theorem 3.7. Let $u$ be a nonnegative solution of $L u=0$ in $Q=\Omega \times(0, T)$ which continuously vanishes on $\partial_{x} Q$, and let $\delta=$ const $>0$. Then there exists a positive constant $N=N\left(n, \nu, m, r_{0}\right.$, $\left.\operatorname{diam} \Omega, T, \delta\right)$, such that

$$
u(x, s) \leq N u(x, t)
$$

where $T>s \geq t \geq s-d^{2} \geq \delta^{2}=$ const $>0, d=\operatorname{dist}(x, \partial \Omega)$. 
We first prove an auxiliary result. For given $Y=(y, s), r>0$ and $k>0$, we set

$$
V_{1}=\Omega_{k r} \times\left(s-r^{2}, s\right), \quad V_{2}=\Omega_{2 k r} \times\left(s-4 r^{2}, s\right),
$$

where $\Omega_{k r}=\Omega \cap B_{k r}(y), \Omega_{2 k r}=\Omega \cap B_{2 k r}(y)$. The parabolic boundary of $V_{2}$ is represented in the form $\partial^{\prime} V_{2}=\Gamma_{0} \cup \Gamma_{1} \cup \Gamma_{2}$, where

$$
\begin{aligned}
& \Gamma_{0} \subset Q_{0} \backslash \partial^{\prime} Q_{0}, \quad \Gamma_{1} \subset \partial_{x} Q_{0}, \quad \Gamma_{2} \subset \partial_{t} Q_{0}, \\
& Q_{0}=B_{2 k r}(y) \times\left(s-4 r^{2}, s\right) \text {. }
\end{aligned}
$$

Lemma 3.8. Let $Y=(y, s) \in \bar{Q}$ and let positive constants $r$ and $\gamma$ be given. There exists a constant $k=k(n, \nu, \gamma) \geq 8$ such that, for any nonnegative solution of $L u=0$ in $V_{2}$ which continuously vanishes on $\Gamma_{0}$, from the inequality

$$
M=\sup _{V_{1}} u>(2 k)^{-\gamma} \sup _{\Gamma_{1}} u
$$

it follows

$$
\sup _{\Gamma_{2}} u>\frac{1}{2} M
$$

Proof. Using the transformations:

$$
x \longrightarrow(2 r)^{-1}(x-y), t \longrightarrow(2 r)^{-2}(t-s)+1 \text {, and } u \longrightarrow \text { const } \cdot u,
$$

we reduce the proof to the case

$$
y=0, \quad s=1, \quad r=\frac{1}{2}, \sup _{\Gamma_{1}} u=1,
$$

so that $V_{1}=\Omega_{\frac{k}{2}} \times\left(\frac{3}{4}, 1\right), \quad V_{2}=\Omega_{k} \times(0,1)$. Next, we show that for the proof of the lemma it suffices to construct a function $v(x, t)$ satisfying the inequalities

$$
v \geq 0, \quad L v \leq 0 \text { in } V_{2}, v \leq N e^{-\frac{k^{2}}{N}} \text { in } V_{1} \text {, and } v \geq 1 \text { on } \Gamma_{1}
$$

with constants $N=N(n, \nu)>0$. Indeed, then we have

$L(u-v)=-L v \geq 0$ in $V_{2}$, and $u-v \leq 0$ on $\Gamma_{0} \cup \Gamma_{1}=\partial^{\prime} V_{2} \backslash \Gamma_{2}$. Therefore,

$$
\begin{gathered}
\sup _{\Gamma_{2}} u \geq \sup _{\Gamma_{2}}(u-v)=\sup _{\partial^{\prime} V_{2}}(u-v) \\
=\sup _{V_{2}}(u-v) \geq \sup _{V_{1}}(u-v) \geq M-N e^{-\frac{k^{2}}{N}},
\end{gathered}
$$

where $N=N(n, \nu)>0$. We can choose $k=k(n, \nu, \gamma) \geq 8$ such that

$$
N e^{-\frac{k^{2}}{N}} \leq \frac{1}{2}(2 k)^{-\gamma}=\frac{1}{2}(2 k)^{-\gamma} \sup _{\Gamma_{1}} u
$$

This gives us

$$
\sup _{\Gamma_{2}} u \geq M-\frac{1}{2}(2 k)^{-\gamma} \sup _{\Gamma_{1}} u .
$$

This inequality together with (24) yields (25). So it remains to construct $v(x, t)$ satisfying (26).

Consider the function

$$
v=k_{0} s^{-\alpha} e^{\frac{\beta|x|^{2}}{s}}, \quad \text { where } s=2-t, \quad k_{0}=2^{\alpha} e^{-\frac{\beta k^{2}}{2}},
$$


and $\alpha, \beta$ are constants. We have $v \geq 0$,

$$
\begin{aligned}
L v & =\sum_{i, j} a_{i j} D_{i j} v+v_{s} \\
& =\frac{v}{s}\left(2 \beta \sum_{i} a_{i i}-\alpha\right)+\frac{\beta v}{s^{2}}\left(4 \beta \sum_{i, j} a_{i j} x_{i} x_{j}-|x|^{2}\right) \leq 0
\end{aligned}
$$

in $V_{2}$ for some $\alpha, \beta>0$ depending only on $n, \nu$. Further,

$$
|x|<\frac{k}{2}, \quad v \leq k_{0} s^{-\alpha} e^{\frac{\beta k^{2}}{4 s}} \leq 2^{\alpha} e^{-\frac{\beta k^{2}}{4}} \leq N e^{-\frac{k^{2}}{N}} \text { in } V_{1} .
$$

Finally, $|x|=k$ and $1<s<2$ imply $v \geq 1$ on $\Gamma_{1}$. Hence $v$ satisfies (26), and the proof of the lemma is completed.

Proof of Theorem 3.7. Denote $\rho_{0}=\frac{1}{2} \min \left(r_{0}, \delta_{0}\right)>0$. If $d>\rho_{0}$, then (21) follows directly from (20) with $\delta=\rho_{0}$, so we may assume $d \leq \rho_{0}$.

We choose $y \in \partial \Omega$ such that $|x-y|=d$, set $Y=(y, s)$, and consider the function

$$
f(\rho)=\rho^{-\gamma} \sup _{Q_{\rho}} u, \quad \text { where } Q_{\rho}=Q \cap C_{\rho}(Y),
$$

and $\gamma=\gamma(n, \nu, m)>0$ is the constant in Lemma 3.2. Now we define

$$
r=\max \left\{\rho: d \leq \rho \leq \rho_{0}, f(\rho) \geq f(d)\right\} .
$$

The inequality $f(d) \leq f(r)$ implies

$$
u(x, s) \leq \sup _{Q_{d}} u \leq\left(\frac{d}{r}\right)^{\gamma} \sup _{Q_{r}} u
$$

By Lemma 3.2 we also have

$$
u\left(\underline{Y}_{r}\right) \leq N\left(\frac{r}{d}\right)^{\gamma} u(x, t) .
$$

These two estimates reduce the proof of (21) to the inequality

$$
M_{1}=\sup _{Q_{r}} u \leq N u\left(\underline{Y}_{r}\right) .
$$

In the proof of this inequality, we consider separately two cases (a) and (b).

(a) $\frac{\rho_{0}}{2 k} \leq r \leq \rho_{0}$, where $k$ is a constant in Lemma 3.8 corresponding to our $\gamma$. Since $r$ is comparable with $\rho_{0}$, from Theorem 3.6 it follows $u\left(\bar{Y}_{r}\right) \leq N u\left(\underline{Y}_{r}\right)$. This estimate and (12) yield (27).

(b) $d \leq r<\frac{\rho_{0}}{2 k}$. By definition of $r, f(r)>f(2 k r)$; hence

$$
M_{1}=\sup _{Q_{r}} u>(2 k)^{-\gamma} \sup _{Q_{2 k r}} u \text {. }
$$

It is easy to see that $Q_{r} \subset V_{1}, \overline{Q_{2 k r}} \supset \Gamma_{1}$, where $V_{1}, \Gamma_{1}$ are defined in (22), (23). Therefore, (28) implies (24) and (25). The last estimate means

$$
M_{1}<2 u\left(Z_{0}\right) \text { for some } Z_{0}=\left(z_{0}, s-4 r^{2}\right), z_{0} \in \overline{\Omega_{2 k r}} .
$$

If $\operatorname{dist}\left(z_{0}, \partial \Omega\right)<r$, then $\left|z_{0}-z\right|<r$ for some $z \in \partial \Omega$, and by (12)

$$
u\left(Z_{0}\right) \leq \sup _{Q_{r}(Z)} u \leq N u\left(\bar{Z}_{r}\right)
$$


The Harnack inequality (5) guarantees $u\left(\bar{Z}_{r}\right) \leq N u\left(\underline{Y}_{r}\right)$, where by "scaling" arguments $N$ depends only on $n, \nu, m$. Therefore,

$$
u\left(Z_{0}\right) \leq N u\left(\underline{Y}_{r}\right) .
$$

If $\operatorname{dist}\left(z_{0}, \partial \Omega\right) \geq r$, then we can use the Harnack inequality directly to get (30). Finally, (29) and (30) provide the estimate (27).

\section{Hölder Continuity of Quotients}

We now begin the discussion leading to the proof of the second main result: the quotient of two solutions of a parabolic non-divergence equation, both solutions vanishing on a portion of the lateral boundary, is locally Hölder continuous up to that portion of the boundary. In this section we need some further Notation: for $Y=(y, s) \in \mathbb{R}^{n+1}$ with $y \in \partial \Omega, 0<r \leq R$, we set

$$
\begin{aligned}
\Omega_{R}(y) & =B_{R}(y) \cap \Omega, \\
\Omega_{R, r}(y) & =\Omega_{R}(y) \cap\{x \in \Omega: d(x)<r\}, \\
Q_{R}(Y) & =Q_{R}(y, s)=\Omega_{R}(y) \times\left(s-R^{2}, s\right), \\
Q_{R, r}(Y) & =Q_{R, r}(y, s)=\Omega_{R, r}(y) \times\left(s-R^{2}, s\right), \\
S_{R, r}(Y) & =\left\{(x, t) \in \partial^{\prime} Q_{R, r}(Y): d(x)=r\right\}, \\
\Gamma_{R, r}(Y) & =\left\{(x, t) \in \partial^{\prime} Q_{R, r}(Y): 0<d(x)<r\right\},
\end{aligned}
$$

where $d(x)=\operatorname{dist}(x, \partial \Omega)$.

4.1. Some Lemmas. We first present two auxiliary lemmas, which are interesting for their own sake.

Lemma 4.1. Let $Y=(y, s) \in \mathbb{R}^{n+1}$ with $y \in \partial \Omega, r>0, K \geq 6, K r \leq r_{0}$, and let $u$ be a solution of $L u \geq 0$ in $Q_{K r, r}(Y)$ satisfying the following conditions: $1 . u \leq 1$ in $Q_{K r, r}(Y), 2 . u \leq 0$ on $\left(\partial^{\prime} Q_{K r, r}\right) \backslash \Gamma_{K r, r}$. Then we have

$$
\sup _{Q_{r}(Y)} u \leq e^{-N K},
$$

where $N=N(n, \nu, m)>0$.

Proof. We prove this decay estimate by iteration. By scaling $x \rightarrow r^{-1} x, t \rightarrow r^{-2} t$, we may assume $r=1$. Let $j \geq 1,2 j+1 \leq K$, $\sup _{Q_{2 j-1,1}} u=u\left(X_{j}\right)$ for some $X_{j} \in \partial^{\prime} Q_{2 j-1,1}$. Since $\partial \Omega$ satisfies the Lipschitz condition, we may apply Lemma 3.4 with a constant $\varepsilon=\varepsilon(n, \nu, m) \in(0,1)$ to $U=C_{2}\left(X_{j}\right) \cap Q_{K, 1} \subset Q_{2 j+1,1}$, then we have

$$
\sup _{Q_{2 j-1,1}} u=u\left(X_{j}\right) \leq \theta \sup _{U} u \leq \theta \sup _{Q_{2 j+1,1}} u,
$$

where $\theta=\theta(n, \nu, m) \in(0,1)$. Notice that $Q_{1,1}=Q_{1}$. Iterating the above estimate, we obtain

$$
\sup _{Q_{1}} u \leq \theta^{k} \sup _{Q_{2 k+1,1}} u \leq \theta^{k}
$$

where $2 k+1 \leq K \leq 2 k+3$. Since $k>\frac{K-3}{2} \geq \frac{K}{4}$, we get the desired estimate (31) with $N=N(n, \nu, m)=-\frac{1}{4} \ln \theta>0$. 
Lemma 4.2. Let $Y=(y, s) \in \partial \Omega \times \mathbb{R}^{1}$ be fixed, and let $u, v$ satisfy the conditions

$$
\begin{aligned}
& \text { 1. } L u \leq 0, \quad u \geq 0 \quad \text { in } \quad Q_{K r, r}, \quad u \geq 1 \quad \text { on } \quad S_{K r, r}, \\
& \text { 2. } L v \geq 0, \quad v \leq 1 \quad \text { in } Q_{K r, r}, \quad v \leq 0 \quad \text { on } \quad\left(\partial^{\prime} Q_{K r, r}\right) \backslash \Gamma_{K r, r},
\end{aligned}
$$

where $K \geq 6,0<K r \leq r_{0}$. Then we have

$$
v \leq u \quad \text { in } \quad Q_{r}=Q_{r}(Y),
$$

provided $K=K(n, \nu, m)$ is large enough.

Proof. As before, we may assume $r=1$. First we prove the estimate

$$
u(X)=u(x, t) \geq 2 \delta d^{\gamma}(x) \text { in } Q_{1}=Q_{1}(Y)
$$

with positive constants $\delta$ and $\gamma$ depending only on $n, \nu, m$.

We choose $R=R(m) \geq 6$ and $\tilde{y} \in \Omega$, such that $|\tilde{y}-y|=R$ and $B_{2}(\tilde{y}) \subset \Omega_{R+2}=$ $\Omega \cap B_{R+2}(y)$, and assume $K \geq R$. Next, we define $\tilde{u}$ in $Q_{R}=Q \cap C_{R}(Y)$ as the (unique) solution of the equation $L \tilde{u}=0$ in $Q_{R}$ with the boundary values

$$
\tilde{u}=\min (u, 1) \quad \text { on } \quad\left(\partial^{\prime} Q_{R}\right) \cap\left(\partial^{\prime} Q_{R, 1}\right), \quad \tilde{u}=1 \quad \text { on } \quad\left(\partial^{\prime} Q_{R}\right) \backslash\left(\partial^{\prime} Q_{R, 1}\right) .
$$

By the comparison principle, $0 \leq \tilde{u} \leq 1$ in $Q_{R}$. Moreover, since

$$
u \geq 1 \quad \text { on } \quad S_{K, 1} \supseteq S_{R, 1} \supseteq\left(\partial^{\prime} Q_{R, 1}\right) \backslash\left(\partial^{\prime} Q_{R}\right),
$$

we have $u \geq \tilde{u}$ on $\partial^{\prime} Q_{R, 1}$, and hence $u \geq \tilde{u}$ in $Q_{R, 1} \supseteq Q_{1}$. Further, we set

$$
z=\tilde{y}+R^{-1}(\tilde{y}-y), \quad \tilde{z}=\tilde{y}-R^{-1}(\tilde{y}-y), \quad \tilde{Y}=(\tilde{y}, s-4), \quad \tilde{Z}=(\tilde{z}, s-4) .
$$

It is easy to see that

$$
B_{1}(z) \subset B_{2}(\tilde{y}) \backslash \Omega_{R}, \quad B_{1}(\tilde{z}) \subset B_{2}(\tilde{y}) \cap \Omega_{R} .
$$

We can apply Lemma 3.4 to the function $1-\tilde{u}$ in $U=Q_{R}(Y) \cap C_{2}(\tilde{Y})$, which vanishes on $\left(\partial^{\prime} Q_{R}\right) \backslash\left(\partial^{\prime} Q_{R, 1}\right) \supseteq\left(\partial^{\prime} U\right) \backslash\left(\partial^{\prime} C_{2}(\tilde{Y})\right)$. This gives us

$$
1-\tilde{u}(\tilde{Z}) \leq \sup _{U \cap C_{1}(\tilde{Y})}(1-\tilde{u}) \leq \theta \sup _{U}(1-\tilde{u}) \leq \theta=\theta(n, \nu)<1,
$$

and $\tilde{u}(\tilde{Z}) \geq 1-\theta>0$. By the Harnack principle, Theorem 2.2,

$$
\tilde{u}\left(\underline{Y}_{1}\right) \geq N^{-1} \tilde{u}(\tilde{Z}) \geq \delta_{0}=\delta_{0}(n, \nu, m)>0 .
$$

Now applying Lemma 3.2 to $\tilde{u}$ in $Q_{R} \supset Q_{2} \supset Q_{1}$, we have

$$
\tilde{u}(X)=\tilde{u}(x, t) \geq N^{-1} d^{\gamma}(x) \tilde{u}\left(\underline{Y}_{1}\right) \geq 2 \delta d^{\gamma}(x) \text { in } Q_{1},
$$

where $\gamma=\gamma(n, \nu, m)>0, \delta=\delta(n, \nu, m)>0$. Since $u \geq \tilde{u}$ in $Q_{1}$, the estimate (34) is proved for $K \geq R$. In particular, for such $K$ we have

$$
u \geq 2 \delta K^{-\gamma} \quad \text { in } \overline{Q_{1} \backslash Q_{1, K^{-1}}} \supset S_{1, K^{-1}} .
$$

It follows from Lemma 4.1 that

$$
v \leq e^{-N K} \leq \delta K^{-\gamma} \quad \text { in } \quad Q_{1},
$$

provided $K=K(n, \nu, m)$ is chosen large enough. Then

$$
\begin{aligned}
& u_{1}=\frac{K^{\gamma}}{2 \delta} u \geq 0 \quad \text { in } \quad Q_{1, K^{-1}}, \quad u_{1} \geq 1 \quad \text { in } \overline{Q_{1} \backslash Q_{1, K^{-1}}} \supset S_{1, K^{-1}}, \\
& v_{1}=\frac{K^{\gamma}}{2 \delta}(2 v-u) \leq \frac{K^{\gamma}}{\delta} v \leq 1 \quad \text { in } \quad Q_{1} \supset Q_{1, K^{-1}}, \quad v_{1} \leq 0 \quad \text { on } \quad S_{1, K^{-1}},
\end{aligned}
$$


and hence

$$
u_{1}-v_{1}=\frac{K^{\gamma}}{\delta}(u-v) \geq 0 \quad \text { in } \quad \overline{Q_{1} \backslash Q_{1, K^{-1}}} .
$$

In particular, $u_{1}, v_{1}$ satisfy the same assumption as (32) with $r=K^{-1}$. By iteration, we can construct $u_{j}, v_{j}$ such that

$$
u_{j}-v_{j}=\left(\frac{K^{\gamma}}{\delta}\right)^{j}(u-v) \geq 0 \quad \text { in } \overline{Q_{K^{1-j}} \backslash Q_{K^{1-j}, K^{-j}}}
$$

for all $j=1,2,3, \cdots$. As a consequence,

$$
u-v \geq 0 \quad \text { on } \quad I(Y)=\bigcup_{j=1}^{\infty} \overline{Q_{K^{1-j}}(Y) \backslash Q_{K^{1-j}, K^{-j}}(Y)} .
$$

For arbitrary $X_{0}=\left(x_{0}, t_{0}\right) \in Q_{1}=Q_{1}(Y)$, we can take $Y_{0}=\left(y_{0}, t_{0}\right)$ with $y_{0} \in \partial \Omega$ satisfying $d\left(x_{0}\right)=\operatorname{dist}\left(x_{0}, \partial \Omega\right)=\left|x_{0}-y_{0}\right|$. Then $X_{0} \in I\left(Y_{0}\right)$. Moreover, $\left|y_{0}-y\right| \leq\left|x_{0}-y\right|+\left|x_{0}-y_{0}\right|<2 ;$ therefore,

$$
Q_{K, 1}\left(Y_{0}\right) \subset Q_{K+2,1}(Y), \quad S_{K, 1}\left(Y_{0}\right) \subset S_{K+2,1}(Y) .
$$

Replacing $K$ with $K+2$, we conclude $u-v \geq 0$ on $I\left(Y_{0}\right) \ni X_{0}$. Since $X_{0}$ is an arbitrary point in $Q_{1}$, we arrive at (33).

Remark. In terms of the $L$-caloric measure $\omega^{X}$ (see [FGS, p. 540]) corresponding to $L$ and $Q_{K r, r}(Y),(33)$ says

$$
\omega^{X}\left(S_{K r, r}\right) \geq \omega^{X}\left(\Gamma_{K r, r}\right) \quad \text { for } \quad X \in Q_{r}(Y) .
$$

\subsection{Boundedness of Quotients.}

Theorem 4.3. Fix $Y=(y, s) \in \partial \Omega \times(0, \infty)$ with $0<K r<\frac{1}{2} \min \left(r_{0}, \sqrt{s}\right)$, where $K$ is the constant in Lemma 4.2. Assume $u$ and $v$ are two nonnegative solutions of $L u=0$ in $\Omega \times(0, \infty)$, and $v=0$ on $C_{2 K r}(Y) \cap(\partial \Omega \times(0, \infty))$; then

$$
\sup _{Q_{r}(Y)} \frac{v}{u} \leq N(n, \nu, m) \frac{v\left(\bar{Y}_{K r}\right)}{u\left(\underline{Y}_{K r}\right)} .
$$

Proof. By scaling, we may assume $r=1, u\left(\underline{Y}_{K}\right)=v\left(\bar{Y}_{K}\right)=1$. By the boundary Harnack inequality, Theorem 3.3,

$$
v \leq N_{0}(n, \nu, m) \quad \text { in } \quad Q_{K} \supset Q_{K, 1} .
$$

By Lemma 3.2 (or Theorem 2.2),

$$
u \geq \frac{1}{N_{0}(n, \nu, m)} \quad \text { on } \quad S_{K, 1} .
$$

Applying Lemma 4.2 to the functions $u_{0}=N_{0} u$ and $v_{0}=N_{0}^{-1} v-u_{0}$, we get

$$
\sup _{Q_{1}} \frac{v}{u}=N_{0}^{2} \sup _{Q_{1}}\left(\frac{v_{0}}{u_{0}}+1\right) \leq 2 N_{0}^{2}=N(n, \nu, m),
$$

the desired estimate (35).

Remark. The above estimate (35) was first proved in $[\mathrm{G}]$ (Theorem 3.1) for the nondivergence case and $C^{2}$-domains and in [FGS] (Theorem 1.6) for the divergence case and Lipschitz domains. For elliptic equations (divergence and non-divergence), it was proved in [CFMS] (Theorem 1.4), [B] (Theorem 2.1), and [FGMS] (Theorem I.3.7). 
4.3. Oscillation Decay. In the following two theorems, we use the notation

$$
\omega(X, r)=\underset{Q_{r}(X)}{\operatorname{OSC}} \frac{v}{u}, \text { where } Q_{r}(X)=Q \cap C_{r}(X) .
$$

Theorem 4.4. Assume $u$ and $v$ are two strictly positive solutions of $L u=0$ in $Q=\Omega \times(0, \infty)$ and also $u=0$ on $\partial_{x} Q=\partial \Omega \times(0, \infty)$.

(a) Let $X=(x, t) \in Q, t>\delta^{2}=$ const $>0$, and $0<r \leq \frac{1}{2} d(X)$, where $d(X)=\min (d(x), \sqrt{t})$. Then

$$
\omega\left(X, \frac{r}{2}\right) \leq \theta_{0} \omega(X, r),
$$

where $\theta_{0}=\theta_{0}\left(n, \nu, m, r_{0}\right.$, diam $\left.\Omega, \delta\right) \in(0,1)$.

(b) Let $Y=(y, s) \in \partial_{x} Q, s \geq \delta^{2}=$ const $>0$ and $0<K r \leq \frac{1}{2} \min \left(r_{0}, \sqrt{s}\right)$, where $K$ is the constant in Lemma 4.2. Let $v=0$ on $C_{K r}(Y) \cap \partial_{x} Q$. Then

$$
\omega\left(Y, \frac{r}{2 K}\right) \leq \theta_{1} \omega(Y, r)
$$

where $\theta_{1}=\theta_{1}\left(n, \nu, m, r_{0}\right.$, diam $\left.\Omega, \delta\right) \in(0,1)$.

Proof. (a) Denote $X^{ \pm}=\left(x, t \pm r^{2} / 2\right)$. We may assume

$$
0 \leq \frac{v}{u} \leq 1=\omega(X, r)=\underset{C_{r}(X)}{\operatorname{osc}} \frac{v}{u} \text { in } C_{r}(X), \text { and } \frac{v}{u}\left(X^{-}\right) \geq \frac{1}{2} ;
$$

otherwise in place of $v$ we take $c_{1} u+c_{2} v$ with some constants $c_{1}, c_{2}$. By the Harnack principle (Theorem 2.2),

$$
v\left(X^{-}\right) \leq N v, \quad u \leq N u\left(X^{+}\right) \text {in } C_{\frac{r}{2}}(X) .
$$

Moreover, by the boundary backward Harnack inequality (Theorem 3.7),

$$
u\left(X^{+}\right) \leq N_{1} u\left(X^{-}\right) .
$$

Thus

$$
\frac{1}{2} \leq \frac{v}{u}\left(X^{-}\right) \leq N_{2} \frac{v}{u} \leq N_{2} \text { in } C_{\frac{r}{2}}(X),
$$

which implies (36) with $\theta_{0}=1-\frac{1}{2 N_{2}}$.

(b) According to Theorem 4.3, $\omega(Y, r)<\infty$. As before, we may assume

$$
0 \leq \frac{v}{u} \leq 1=\omega(Y, r) \quad \text { in } \quad Q_{r}(Y), \text { and } \frac{v}{u}\left(\underline{Y}_{\frac{r}{2}}\right) \geq \frac{1}{2} .
$$

Applying Theorem 4.3 again and then Theorem 3.7, we get

$$
\sup _{Q_{\frac{r}{2 K}}} \frac{u}{v} \leq N \frac{u\left(\bar{Y}_{\frac{r}{2}}\right)}{v\left(\underline{Y}_{\frac{r}{2}}\right)} \leq N_{3} \frac{u}{v}\left(\underline{Y}_{\frac{r}{2}}\right) \leq 2 N_{3}=2 N_{3}\left(n, \nu, m, r_{0}, \operatorname{diam} \Omega, \delta\right) .
$$

Thus

$$
\frac{1}{2 N_{3}} \leq \frac{v}{u} \leq 1 \text { in } Q_{\frac{r}{2 K}}(Y)
$$

which implies (37) with $\theta_{1}=1-\frac{1}{2 N_{1}}$.

Theorem 4.5. Let $u$ and $v$ be two strictly positive solutions of $L u=0$ in $Q=$ $\Omega \times(0, \infty)$ such that $u=0$ on $\partial_{x} Q=\partial \Omega \times(0, \infty)$, and $v=0$ on $C_{K r_{1}}(Y) \cap \partial_{x} Q$, where $Y=(y, s) \in \partial_{x} Q, s \geq \delta^{2}=$ const $>0, K$ is the constant in Lemma 4.2, and

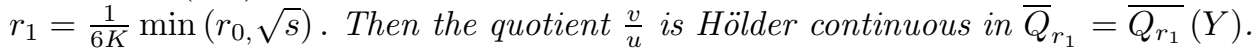


Proof. By Theorem 4.3, we have

$$
\omega\left(Y, 3 r_{1}\right) \leq N \frac{v\left(\bar{Y}_{3 K r_{1}}\right)}{u\left(\underline{Y}_{3 K r_{1}}\right)}=N_{0}<\infty .
$$

For arbitrary $X=(x, t) \in \bar{Q}_{r_{1}}$ and $0<r \leq r_{1}$, we deal with three cases:

(a) $0<r \leq d \leq r_{1}$, (b) $0 \leq d<r \leq \frac{1}{2} r_{1}$, and (c) $d<r, \frac{1}{2} r_{1}<r \leq r_{1}$, where $d=d(x)=\operatorname{dist}(x, \partial \Omega)$.

Case (a). Iterating (36), we get

$$
\omega(X, r) \leq 2^{\alpha_{0}}\left(\frac{2 r}{d}\right)^{\alpha_{0}} \omega\left(X, \frac{d}{2}\right) \text { for } 0<r \leq \frac{d}{2},
$$

where $\alpha_{0}=-\log _{2} \theta_{0}>0$. This implies the estimate

$$
\omega(X, r) \leq\left(\frac{4 r}{d}\right)^{\alpha_{0}} \omega(X, d),
$$

which is also true for $\frac{d}{2}<r \leq d$.

Case (b). In this case, $Q_{r}(X) \subset Q_{2 r}\left(X_{0}\right)$ for some $X_{0}=\left(x_{0}, t\right) \in \partial_{x} Q$ with $\left|x-x_{0}\right|=d=d(x)$. By iterating estimate (37), we have

$$
\omega(X, r) \leq \omega\left(X_{0}, 2 r\right) \leq\left(\frac{4 K r}{r_{1}}\right)^{\alpha_{1}} \omega\left(X_{0}, r_{1}\right) \quad \text { for } \quad d \leq r \leq \frac{1}{2} r_{1},
$$

where $\alpha_{1}=-\log _{2 K} \theta_{1}>0$.

Combining (39) and (40), we get

$\omega(X, r) \leq\left(\frac{4 r}{d}\right)^{\alpha_{0}} \omega(X, d) \leq\left(\frac{4 r}{d}\right)^{\alpha_{0}}\left(\frac{4 K d}{r_{1}}\right)^{\alpha_{1}} \omega\left(X_{0}, r_{1}\right) \quad$ for $0<r \leq d \leq \frac{1}{2} r_{1}$.

Notice that $Q_{r_{1}}\left(X_{0}\right) \subset Q_{2 r_{1}}(X) \subset Q_{3 r_{1}}(Y)$; hence by virtue of (38), $\omega\left(X_{0}, r_{1}\right) \leq$ $\omega\left(Y, 3 r_{1}\right) \leq N_{0}$. We set $\alpha=\min \left(\alpha_{0}, \alpha_{2}\right)$. We may assume that the constants $\theta_{0}, \theta_{1}$ are close to 1 , so that $\alpha_{0}, \alpha_{1}, \alpha \in(0,1)$. Then the above estimate gives us

$$
\omega(X, r) \leq 16 K\left(\frac{r}{r_{1}}\right)^{\alpha} N_{0}
$$

for arbitrary $X \in \bar{Q}_{r_{1}}$ and $0<r \leq d \leq \frac{1}{2} r_{1}$. If $0<r \leq \frac{1}{2} r_{1}<d \leq r_{1}$, it is a consequence of (39). If $d<r \leq \frac{1}{2} r_{1}$, this estimate follows immediately from (40). In case (c) and the remaining of case (a), we have $r>\frac{1}{2} r_{1}$, which also implies (41). Thus the estimate (41) holds for all $X \in \bar{Q}_{r_{1}}, 0<r \leq r_{1}$, and this provides the Hölder continuity of the quotient $\frac{v}{u}$.

Finally, we are ready to prove the Hölder continuity of the quotient of $\frac{v}{u}$ with $u$ and $v$ vanishing on an open portion of the lateral boundary.

Theorem 4.6. Let $u$ and $v$ be strictly positive solutions of $L u=0$ in $Q=\Omega \times$ $(0, \infty)$, vanishing on $C_{2 r}\left(Y_{0}\right) \cap \partial_{x} Q$, where $Y_{0}=\left(y_{0}, s_{0}\right) \in \partial_{x} Q=\partial \Omega \times(0, \infty)$ and $s_{0} \geq 4 r^{2}>0$. Then $\frac{v}{u}$ is Hölder continuous in $\overline{Q_{r}}\left(Y_{0}\right)$.

Proof. First we assume $u \equiv 0$ on $\partial_{x} Q$. The Hölder continuity of $u$ and $v$ in any subdomain $Q^{\prime} \subset \overline{Q^{\prime}} \subset Q$, which is known from [KS], implies the same property for $\frac{v}{u}$ (it can also be obtained by iteration of (39)). Moreover, by Theorem 4.5, $\frac{v}{u}$ is Hölder continuous in $\overline{Q_{r_{1}}}(Y)$ for all $Y \in C_{r}\left(Y_{0}\right) \cap \partial_{x} Q$ and small $r_{1}>0$. Combining these two facts, we get the Hölder continuity of $\frac{v}{u}$ in $\overline{Q_{r}}\left(Y_{0}\right)$. 
In the general case, as in [FS], we represent $u$ in the form $u=u_{0}+u_{1}$, where

$$
\begin{aligned}
L u_{0} & =L u_{1}=0 \quad \text { in } Q \\
u_{0} & =0, \quad u_{1}=u \quad \text { on } \partial_{x} Q, \\
u_{0} & =u, \quad u_{1}=0 \quad \text { on } \partial_{t} Q=\bar{\Omega} \times\{0\} .
\end{aligned}
$$

Here without loss of generality we may assume $u>0$ on $\Omega \times\{0\}$; otherwise we replace $t$ by $t+$ const. Then $u_{0}>0$ in $Q$, and the previous arguments show that $\frac{v}{u_{0}}$ and $\frac{u_{1}}{u_{0}}$ are Hölder continuous in $\overline{Q_{r}}\left(Y_{0}\right)$. Hence the same holds true for

$$
\frac{v}{u}=\frac{v}{u_{0}} \cdot \frac{1}{1+\frac{u_{1}}{u_{0}}} .
$$

This completes the proof.

Remark. The similar result for divergence case in [FS] (Theorem 8) was obtained by employing Green's function. Our proof of Theorem 4.4 also works for the divergence case, since the boundary backward Harnack inequality is available (Theorem 4 in $[\mathrm{FS}])$.

\section{REFERENCES}

[AC] I. Athanasopoulos and L. A. Caffarelli, A theorem of real analysis and its application to free boundary problems, Comm. Pure and Appl. Math., 38(1985), 499-502. MR 86j:49062

[ACS] I. Athanasopoulos, L. A. Caffarelli and S. Salsa, Caloric functions in Lipschitz domains and regularity of solutions to phase transition problems, Ann. Math., 143(1996), 413-434. MR 97e:35074

[B] P. E. Bauman, Positive solutions of elliptic equations in non-divergence form and their adjoints, Arkiv fur Mathematik, 22(1984), 153-173. MR 86m:35008

[CFMS] L. A. Caffarelli, E. B. Fabes, S. Mortola and S. Salsa, Boundary behavior of nonnegative solutions of elliptic operators in divergence form, Indiana J. of Math., 30(1981), 621-640. MR 83c:35040

[FGMS] E. B. Fabes, N. Garofalo, S. Marín-Malave and S. Salsa, Fatou theorems for some nonlinear elliptic equations, Revista Math. Iberoamericana, 4(1988), 227-251. MR 91e:35092

[FGS] E. B. Fabes, N. Garofalo and S. Salsa, A backward Harnack inequality and Fatou theorem for nonnegative solutions of parabolic equations, Illinois J. of Math., 30(1986), 536-565. MR 88d:35089

[FK] E. B. Fabes and C. E. Kenig, Examples of singular parabolic measures and singular transition probability densities, Duke Math. J., 48(1981), 845-856. MR 86j:35081

[FS] E. B. Fabes and M. V. Safonov, Behavior near boundary of positive solutions of second order parabolic equations, J. Fourier Anal. and Appl., Special Issue: Proceedings of the Conference El Escorial 96, 3(1997), 871-882. MR 99d:35071

[FSt] E. B. Fabes and D. W. Stroock, A new proof of Moser's parabolic Harnack inequality using the old idea of Nash, Arch. Rational Mech. Anal., 96(1986), 327-338. MR 88b:35037

[G] N. Garofalo, Second order parabolic equations in nonvariational form: boundary Harnack principle and comparison theorems for nonnegative solutions, Ann. Mat. Pura Appl., 138(1984), 267-296. MR 87f:35115

[JK] D. S. Jerison and C. E. Kenig, Boundary behavior of harmonic functions in nontangentially accessible domains, Adv. in Math., 46(1982), 80-147. MR 84d:31005b

[K] N. V. Krylov, Nonlinear Elliptic and Parabolic Equations of Second Order, Nauka, Moscow, 1985 in Russian; English transl.: Reidel, Dordrecht, 1987. MR 88d:35005

[KS] N. V. Krylov and M. V. Safonov, A certain property of solutions of parabolic equations with measurable coefficients, Izvestia Akad. Nauk SSSR, ser. Matem., 44(1980), 161-175 in Russian; English transl. in Math. USSR Izvestija, 16(1981), 151-164. MR 83c:35059

[LSU] O. A. Ladyzhenskaya, V. A. Solonnikov and N. N. Ural'tseva, Linear and quasi-linear equations of parabolic type, Nauka, Moscow, 1967 in Russian; English transl.: Amer. Math. Soc., Providence, RI, 1968. MR 39:3159a 
[M1] J. Moser, A Harnack inequality for parabolic differential equations, Comm. Pure and Appl. Math., 17(1964), 101-134; and correction in: Comm. Pure and Appl. Math., 20(1967), 231-236. MR 34:6288

[M2] J. Moser, On a pointwise estimate for parabolic differential equations, Comm. Pure and Appl. Math., 24(1971), 727-740. MR 44:5603

[S] M. V. Safonov, Abstracts of Communications, Third Vilnius conference on probability theory and mathematical statistics, June 22-27, 1981.

[SY] M. V. Safonov and Yu Yuan, Doubling properties for second order parabolic equations, to appear in Ann. Math.

[Sl] S. Salsa, Some properties of nonnegative solutions of parabolic differential operators, Ann. Mat. Pura Appl., 128(1981), 193-206. MR 83j:35078

[T] N. S. Trudinger, Pointwise estimates and quasilinear parabolic equations, Comm. Pure and Appl. Math., 21(1968), 205-226.

School of Mathematics, University of Minnesota, Minneapolis, Minnesota 55455-0100

E-mail address: safonov@math.umn.edu

School of Mathematics, University of Minnesota, Minneapolis, Minnesota 55455-0100

Current address: Department of Mathematics, University of Texas at Austin, Austin, Texas 78712

E-mail address: yyuan@math.utexas.edu 